\title{
Circulating circRNA as biomarkers for dilated cardiomyopathy etiology
}

\author{
Marina C. Costa ${ }^{1} \cdot$ Maria Calderon-Dominguez ${ }^{2}$ - Alipio Mangas ${ }^{2,3,4} \cdot$ Oscar Campuzano $^{5,6,7}$. \\ Georgia Sarquella-Brugada ${ }^{5,6,7}$. Mónica Ramos ${ }^{8}$ - Maribel Quezada-Feijoo ${ }^{8}$. José Manuel García Pinilla9,10. \\ Ainhoa Robles-Mezcua ${ }^{9,10}$ - Galan del Aguila Pacheco-Cruz ${ }^{2}$ - Thalia Belmonte ${ }^{2}$. Francisco J. Enguita ${ }^{1} \cdot$ Rocío Toro $^{2,4}$
}

Received: 9 March 2021 / Revised: 22 June 2021 / Accepted: 14 July 2021

(c) The Author(s) 2021

\begin{abstract}
Dilated cardiomyopathy (DCM) is the third most common cause of heart failure. The multidisciplinary nature of testing — involving genetics, imaging, or cardiovascular techniques - makes its diagnosis challenging. Novel and reliable biomarkers are needed for early identification and tailored personalized management. Peripheral circular RNAs (circRNAs), a leading research topic, remain mostly unexplored in DCM. We aimed to assess whether peripheral circRNAs are expressed differentially among etiology-based DCM. The study was based on a case-control multicentric study. We enrolled 130 subjects: healthy controls $(n=20)$, idiopathic DCM $(n=30)$, ischemic DCM $(n=20)$, and familial DCM patients which included pathogen variants of (i) LMNA gene $(n=30)$ and (ii) BCL2-associated athanogene 3 (BAG3) gene $(n=30)$. Differentially expressed circRNAs were analyzed in plasma samples by quantitative RT-PCR and correlated to relevant systolic and diastolic parameters. The pathophysiological implications were explored through bioinformatics tools. Four circRNAs were overexpressed compared to controls: hsa_circ_0003258, hsa_circ_0051238, and hsa_circ_0051239 in LMNA-related DCM and hsa_circ_0089762 in the ischemic DCM cohort. The obtained areas under the curve confirm the discriminative capacity of circRNAs. The circRNAs correlated with some diastolic and systolic echocardiographic parameters with notable diagnostic potential in DCM. Circulating circRNAs may be helpful for the etiology-based diagnosis of DCM as a non-invasive biomarker.
\end{abstract}

Key messages

- The limitations of cardiac diagnostic imaging and the absence of a robust biomarker reveal the need for a diagnostic tool for dilated cardiomyopathy (DCM).

\footnotetext{
Maria Calderon-Dominguez

mariacalderond@gmail.com

Rocío Toro

rociotorogreen@gmail.com
}

Extended author information available on the last page of the article
- The circular RNA (circRNA) expression pattern is paramount for categorizing the DCM etiologies.

- Our peripheral circRNAs fingerprint discriminates between various among etiology-based DCM and correlates with some echocardiographic parameters.

- We provide a potential non-invasive biomarker for the etiology-based diagnosis of LMNA-related DCM and ischemic DCM.

Keywords Circulating circular RNA $\cdot$ Ischemic-dilated cardiomyopathy $\cdot$ Lamin A/C-dilated cardiomyopathy

\section{Introduction}

Heart failure is a global pandemic affecting more than 25 million people worldwide, with a continuously increasing prevalence [1]. One of the major causes of heart failure is dilated cardiomyopathy (DCM), characterized by chamber enlargement and contractile dysfunction of the left ventricle (LV) [2]. Several etiologies are included in the DCM common pathway. Ischemic cardiomyopathy is more common than non-ischemic (59\% compared with 41\%) [2]. Among non-ischemic cardiomyopathy, up to $35 \%$ of idiopathic DCM may have a family history $[2,3]$. Pathogenic alterations in the gene encoding nuclear lamin $\mathrm{A}$ and $\mathrm{C}$ proteins-lamin A/C (LMNA) explain 5-10\% of familial DCM cases.

DCM is a heterogeneous entity that has different outcomes and may require diverse therapies [4]. Notably, ischemic and familial DCM are major groups with life-threatening arrhythmias [3]. $L M N A$-related DCM presents highly aggressive outcomes and lethal ventricular arrhythmias [5]. Male sex, LV ejection fraction (LVEF) lower than 50\%, and non-missense mutations are independent predictors of adverse outcome [6]. Thus, the identification of DCM etiology may help clinicians to stratify patients at risk of fatal events. However, the diagnosis process to reach DCM etiology involves several clinical 
steps. Multidisciplinary teams, imaging tests, the high cost of genetic testing, and its low efficiency make DCM etiologic diagnosis challenging. A precise, accessible biomarker that supports this process is required to improve diagnosis and early identification of asymptomatic cases. This would facilitate the adoption of tailored management.

Non-coding RNAs have pivotal roles in regulating the network that governs the physiology and pathology of cardiovascular diseases [7]. To date, microRNAs (miRNAs) have been considered a more relevant biomarker candidate due to the complexity of circular RNA (circRNA) assessment in human screening [8]. However, circRNAs also have thoughtprovoking features. The advantages of circRNAs are their cell type, tissue, and developmental stage specificity. Furthermore, they are independently regulated and more stable than lineal RNA, and they are gathered in cells and human body fluids [8]. CircRNAs modulate gene expression by sponging miRNAs, interacting with RNA-binding proteins (RBPs), and competing with canonical splicing of their pre-mRNA precursor [9]. Research reporting circRNAs as an effective diagnostic and therapeutic biomarker in many diseases has grown exponentially in the last decade. Nevertheless, the potential of using this easy-to-monitor and highly stable marker for stratifying DCM etiologies remains unexplored. Additionally, the experimental and computational analyses of these molecular crossregulations will propel new insights on DCM [8].
Fig. 1 Flowchart of the study design strategy. This figure illustrates the experimental workflow of the study including screening, validation, and peripheral circRNAs overexpressed for the $L M N A^{\mathrm{Ph}-}$, $L M N A^{\mathrm{Ph}+}$, and ischemic DCM cohort. Abbreviations: BAG3, BCL2-associated athanogene 3; DCM, dilated cardiomyopathy; lamin $\mathrm{A} / \mathrm{C} ; L M N A^{\mathrm{Ph}-}, L M N A$ carrier of the pathogenic variant; $L M N A^{\mathrm{Ph}+}, L M N A$ carrier phenotype positive; LVEF, left ventricle ejection fraction

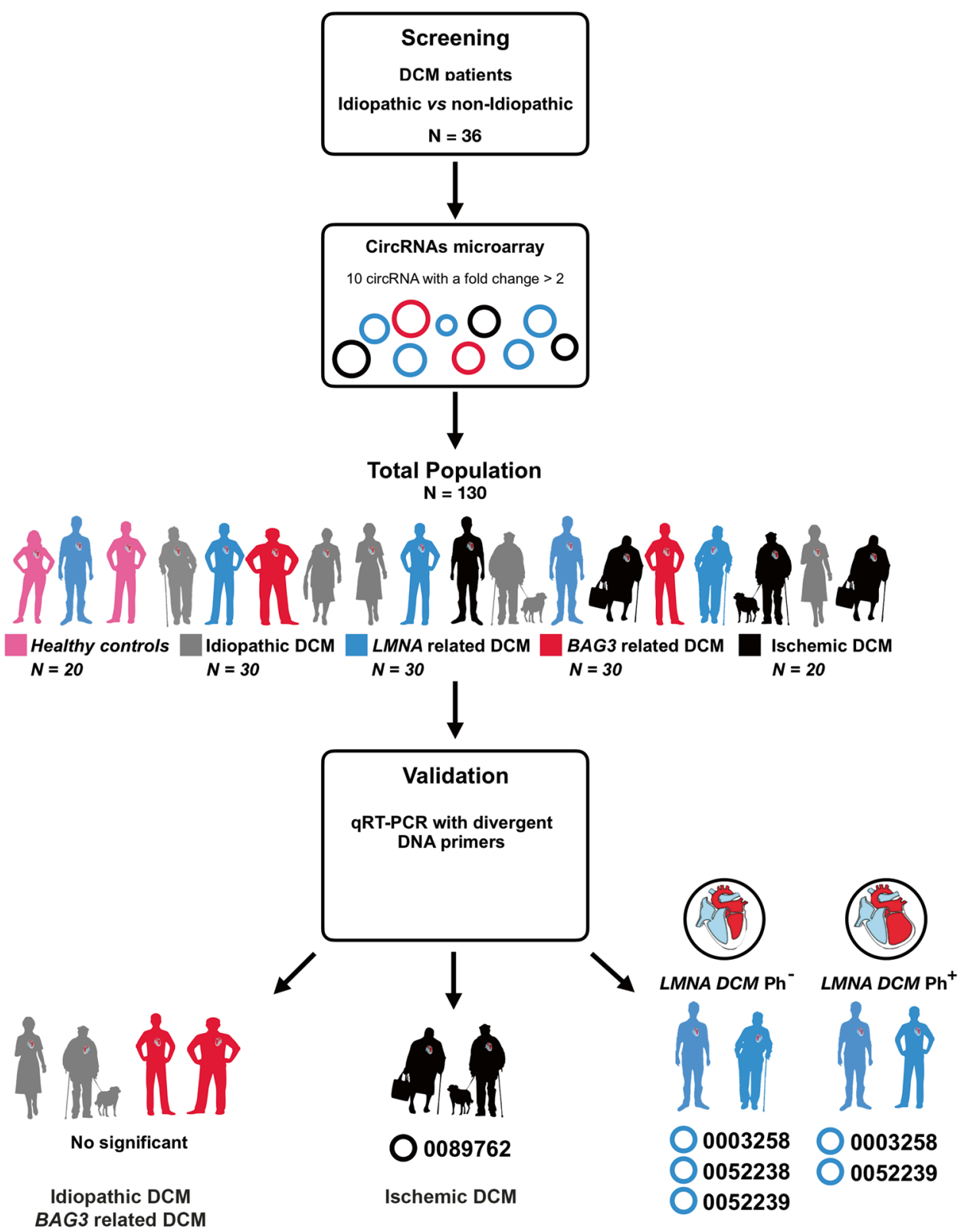


The present study aimed to identify differentially expressed circRNAs in the plasma of patients with DCM of various etiologies such as familial, idiopathic, or ischemic.

\section{Material and methods}

\section{Study design}

The study was based on a case-control multicentric study. Patient samples and the dataset were collected from several centers (Puerta del Mar University Hospital, Cádiz; Cruz Roja Hospital, Madrid; and Virgen de la Victoria University Hospital, Málaga, Spain). We enrolled 130 subjects distributed in five study groups: healthy controls $(n=20)$, idiopathic DCM $(n=30)$, ischemic DCM $(n=20)$, and familial DCM patients. The carriers of rare pathogenic variants included were (i) $L M N A$ gene $(n=30)$ and (ii)
BCL2-associated athanogene 3 (BAG3) gene $(n=30)$ (Fig. 1).

DCM etiology was determined by three independent clinical cardiologists, who are experts in cardiomyopathies. DCM was defined as either LVEF levels below 50\% and/or LV end-diastolic diameter larger than $56 \mathrm{~mm}$ [10]. $B A G 3$ and $L M N A$ participants were confirmed genetically and fulfilled the diagnostic clinical criteria for familial DCM [11]. The LMNA cohort was subclassified as a carrier of the pathogenic variant, phenotypically negative $\left(L M N A^{P h-}\right)$ and genetically and phenotypically positive $\left(L M N A^{\mathrm{Ph}+}\right)$ as previously described [11]. Genetic etiology was ruled out in all idiopathic DCM patients. Ischemic DCM was diagnosed if a precedent of acute myocardial infarction or coronary artery disease was shown, which developed LV remodeling and dysfunction [10]. A transthoracic echocardiography protocol was performed as described previously $[11,12]$. The information included

Table 1 Study population: anthropometric, clinical, and echocardiographic variables

\begin{tabular}{|c|c|c|c|c|c|c|}
\hline Variable & $\begin{array}{l}\text { Healthy } \\
\text { control } \\
(N=20)\end{array}$ & Idiopathic $(N=30)$ & $L M N A^{\mathrm{Ph}-}(N=12)$ & $L M N A^{\mathrm{Ph}+}(N=18)$ & $B A G 3(N=30)$ & Ischemic $(N=20)$ \\
\hline Age (years) & $42.0 \pm 11.0$ & $63.7 \pm 8.2$ & $40.6 \pm 6.9$ & $38.7 \pm 15.0$ & $42.2 \pm 14.8$ & $71.1 \pm 8.5$ \\
\hline Sex (male) & $55 \%$ & $70 \%$ & $23.1 \%$ & $42.9 \%$ & $68.4 \%$ & $72.2 \%$ \\
\hline BMI $\left(\mathrm{kg} / \mathrm{m}^{2}\right)$ & $25.1 \pm 3.3$ & $26.7 \pm 2.6$ & $25.4 \pm 2.1$ & $23.6 \pm 3.9$ & $27.9 \pm 4.9$ & $28.8 \pm 4.9$ \\
\hline Heart rate (bpm) & $65.7 \pm 11.9$ & $71 \pm 13.9$ & $65.7 \pm 5.9$ & $64.3 \pm 9.9$ & $73 \pm 10$ & $64.6 \pm 16.8$ \\
\hline Smoker & $0 \%$ & $60 \%$ & $57.1 \%$ & $30.8 \%$ & $26.3 \%$ & $22.2 \%$ \\
\hline $\mathrm{SBP}(\mathrm{mm} \mathrm{Hg})$ & $114.5 \pm 8.7$ & $113.1 \pm 11.9$ & $128.4 \pm 15.9$ & $123.2 \pm 20.9$ & $128.1 \pm 13.3$ & $124.3 \pm 12.7$ \\
\hline DBP (mm Hg) & $73.5 \pm 8.5$ & $73.1 \pm 7.1$ & $81.8 \pm 6.1$ & $76.7 \pm 17.9$ & $81.1 \pm 7.8$ & $72.2 \pm 8.6$ \\
\hline LVEF (\%) & $68.8 \pm 6.0$ & $30.5 \pm 10.2$ & $44.5 \pm 5.0$ & $61.0 \pm 5.9$ & $49.5 \pm 11.9$ & $34.7 \pm 7.5$ \\
\hline LVEDD (mm) & $47.7 \pm 4.8$ & $63.0 \pm 3.8$ & $58.0 \pm 3.4$ & $49.2 \pm 12.6$ & $55.6 \pm 7.5$ & $58.6 \pm 4.8$ \\
\hline $\operatorname{LVESD}(\mathrm{mm})$ & $30.0 \pm 6.9$ & $48.1 \pm 16.8$ & $43.8 \pm 3.1$ & $30.7 \pm 6.8$ & $40.4 \pm 9.3$ & $44.1 \pm 13.2$ \\
\hline LA volume $\left(\mathrm{mL} / \mathrm{m}^{2}\right)$ & $17.4 \pm 4.3$ & $71.1 \pm 25.0$ & $49.3 \pm 12.4$ & $41.0 \pm 15.5$ & $68.2 \pm 25.8$ & $62.1 \pm 19.6$ \\
\hline LAD (mm) & $35.1 \pm 5.4$ & $45.2 \pm 9.1$ & $40.8 \pm 4.3$ & $33.8 \pm 6.6$ & $37.6 \pm 6.5$ & $40.8 \pm 6.1$ \\
\hline $\mathrm{RV}(\mathrm{mm})$ & $28.6 \pm 3.5$ & $39.7 \pm 6.5$ & $31.7 \pm 1.9$ & $28.8 \pm 5.2$ & $32.1 \pm 7.6$ & $31.4 \pm 6.9$ \\
\hline TAPSE & $22.2 \pm 2.7$ & $18.2 \pm 6.4$ & $21.6 \pm 3.6$ & $21.3 \pm 3.5$ & $21.1 \pm 5.4$ & $18.8 \pm 3.9$ \\
\hline MAPSE & $18.1 \pm 1.6$ & $9.6 \pm 2.7$ & $12.1 \pm 3.1$ & $16.0 \pm 2.6$ & $12.3 \pm 3.2$ & $10.6 \pm 2.1$ \\
\hline $\mathrm{E}(\mathrm{cm} / \mathrm{s})$ & $0.7 \pm 0.2$ & $0.7 \pm 0.2$ & $0.8 \pm 0.1$ & $0.8 \pm 0.2$ & $0.8 \pm 0.3$ & $0.8 \pm 0.2$ \\
\hline $\mathrm{A}(\mathrm{cm} / \mathrm{s})$ & $0.6 \pm 0.1$ & $0.8 \pm 0.3$ & $0.7 \pm 0.3$ & $0.5 \pm 0.2$ & $0.6 \pm 0.2$ & $0.8 \pm 0.3$ \\
\hline S'sTDI (cm/s) & $0.08 \pm 0.01$ & $0.06 \pm 0.06$ & $0.06 \pm 0.01$ & $0.08 \pm 0.02$ & $0.08 \pm 0.01$ & $0.05 \pm 0.01$ \\
\hline E's TDI $(\mathrm{cm} / \mathrm{s})$ & $0.09 \pm 0.03$ & $0.05 \pm 0.05$ & $0.07 \pm 0.02$ & $0.10 \pm 0.04$ & $0.09 \pm 0.04$ & $0.05 \pm 0.01$ \\
\hline A's TDI (cm/s) & $0.10 \pm 0.03$ & $0.06 \pm 0.02$ & $0.11 \pm 0.02$ & $0.09 \pm 0.04$ & $0.11 \pm 0.03$ & $0.07 \pm 0.03$ \\
\hline $\mathrm{E} / \mathrm{E}^{\prime}$ ratio & $7.7 \pm 2.1$ & $16.3 \pm 8.5$ & $10.2 \pm 3.0$ & $7.9 \pm 2.2$ & $6.3 \pm 1.5$ & $15.0 \pm 6.4$ \\
\hline NYHA functional class (II-III) & $0 \%$ & $10 \%$ & $14.3 \%$ & $15.4 \%$ & $10.5 \%$ & $11.1 \%$ \\
\hline
\end{tabular}

All values are expressed as mean \pm SEM

$A$ atrial systolic transmitral flow wave, $A$ 's $T D I$ atrial septal mitral annular velocity, BAG3 BCL2-associated athanogene 3, BMI body mass index, $D B P$ diastolic blood pressure, $D C M$ dilated cardiomyopathy, $E$ early diastolic transmitral flow wave, $E^{\prime}$ early diastolic mitral annular velocity, $L A$ left atrial, $L A D$ left atrial dimension, $L M N A$ lamin A/C, $L M N A^{P h-} L M N A$ carrier of the pathogenic variant, $L M N A^{P h+} L M N A$ carrier phenotypically positive, $L V E D D$ left ventricular end-diastolic dimension, $L V E F$ left ventricle ejection fraction, $L V E S D$ left ventricle end-systolic dimension, MAPSE mitral annular plane systolic excursion, $N Y H A$ New York Heart Association classification, $R V$ right ventricle, $S$ ' positive systolic wave, SBP systolic blood pressure, TAPSE tricuspid annular plane systolic excursion, TDI tissue Doppler imaging 
anthropometric, clinical, therapeutic, electrocardiographic, and echocardiographic data from electronic medical records (Table 1).

\section{Ethics}

The study protocol was approved by the Andalusian Biomedical Research Ethics committee. The study was performed in full compliance with the Declaration of Helsinki. All participants provided written informed consent.

\section{Genetic analysis}

Genetic analysis was performed as previously described [11]. DNA isolation was undertaken using Chemagic MSM I from whole blood (Chemagic Human Blood). DNA integrity was assessed on $0.8 \%$ agarose gel and the quality ratios of absorbance were accomplished using spectrophotometric measurements. dsDNA concentration was determined using fluorometry integrity (Qubit, Life Technologies) and corroborated on $0.8 \%$ agarose gel.

\section{Blood collection}

Ten milliliters of peripheral blood was collected in K2-ethylenediaminetetraacetic acid tubes (BD) after $10 \mathrm{~h}$ overnight fasting. None of the patients was under heparin therapy. The blood was processed within $4 \mathrm{~h}$ after isolation, centrifuged $\left(1500 \mathrm{~g}, 15 \mathrm{~min}, 4{ }^{\circ} \mathrm{C}\right)$, and the plasma layer was aliquoted and stored at $-80^{\circ} \mathrm{C}$ until further analysis.

\section{Microarray analysis}

A screening study was carried out using the Arraystar Human Circular RNA Microarray V2.0 (Arraystar, Inc.). This platform analyzed 36 samples of idiopathic and nonidiopathic DCM subjects. Total RNAs from each sample were obtained using the Arraystar's standard protocols (Arraystar, Inc.). The enriched circRNAs were amplified and transcribed into fluorescent cDNA using a random priming method (Arraystar Super RNA Labeling Kit; Arraystar). The labelled cDNAs were hybridized onto the Arraystar Human circRNA Array V2.0 (Arraystar, Inc.). Once the slides had been washed, they were scanned by the Agilent Scanner G2505C.

\section{RNA isolation and quantitative reverse transcriptase-polymerase chain reaction}

Total RNA was isolated from $200 \mu \mathrm{L}$ of plasma using a miRNeasy Serum/Plasma Kit (Qiagen). RNA was eluted

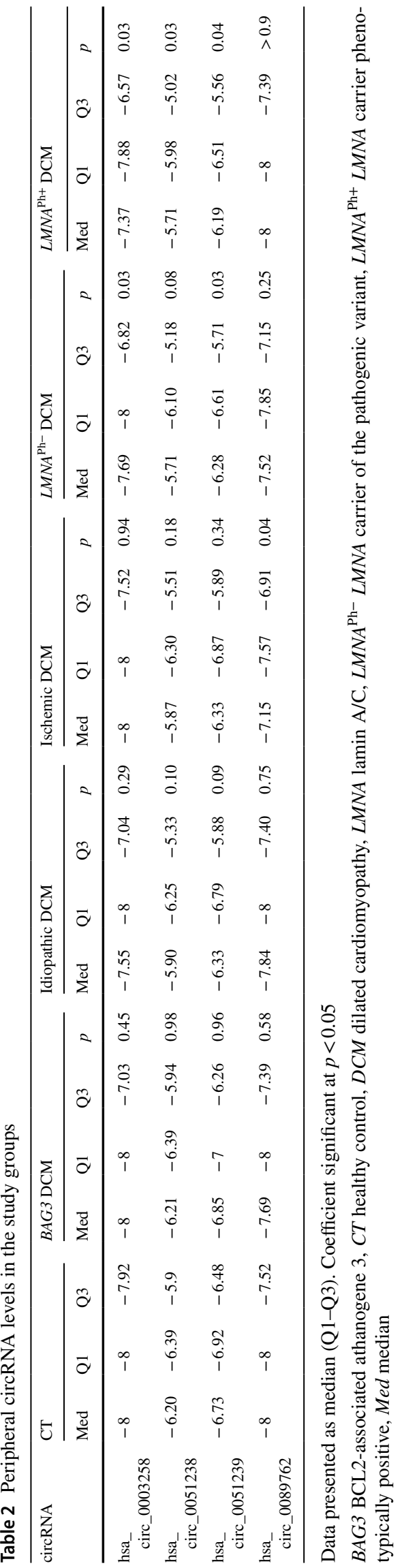




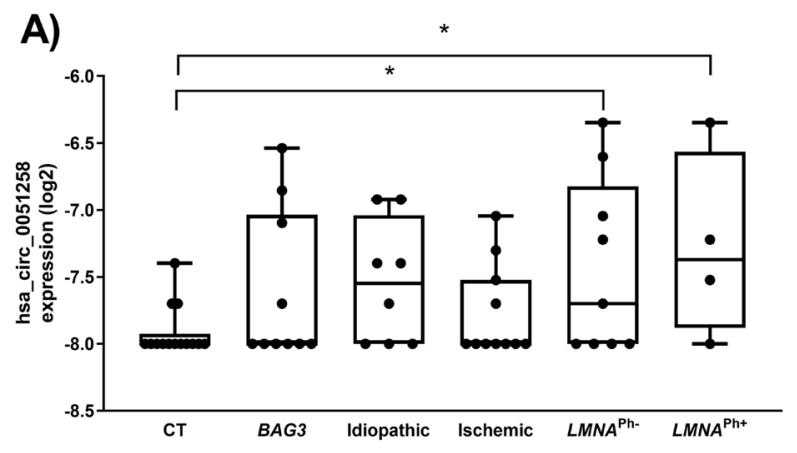

C)

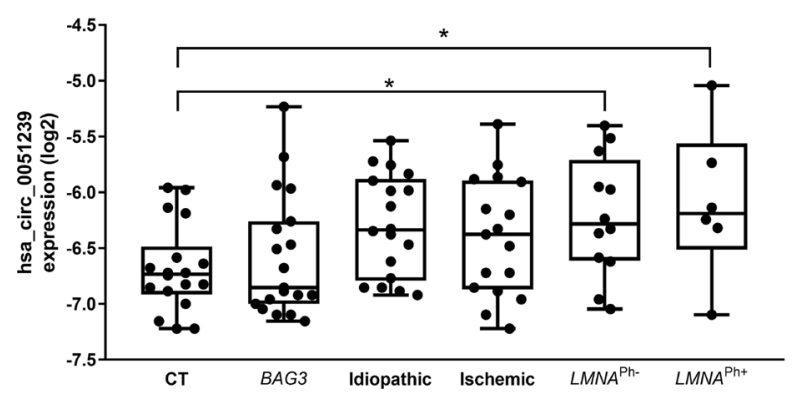

Fig. 2 Boxplots of circRNA expression levels, normalized to MS2 RNA, in healthy subjects, BAG3-related DCM, idiopathic DCM, ischemic DCM, and LMNA-related DCM. The analysis was carried out using qRT-PCR. Data are present in $\log _{2}$. Data represent the mean \pm SEM. $* p<0.05$. Abbreviations: BAG3, BCL2-associated

with $20 \mu \mathrm{L}$ of RNase-free $\mathrm{H}_{2} \mathrm{O}$ and stored at $-80^{\circ} \mathrm{C}$. For the circRNA quantification, circulating RNA preparations were reverse transcribed with a first-strand cDNA synthesis kit (Nzytech, Portugal) using a random primer approach and following the manufacturer's instructions. Previous to reverse transcription, samples were spiked with MS2 RNA (Sigma-Aldrich, Germany), which was used as an internal normalizer. Quantification of selected circRNAs was performed by qRT-PCR using divergent DNA primers designed with the circInteractome algorithm [13] (see Supplemental Table 1 for primer sequences) in an Applied Biosystems by the qRT-PCR system. Fold-change analysis between sample groups was calculated by the Delta-Ct method.

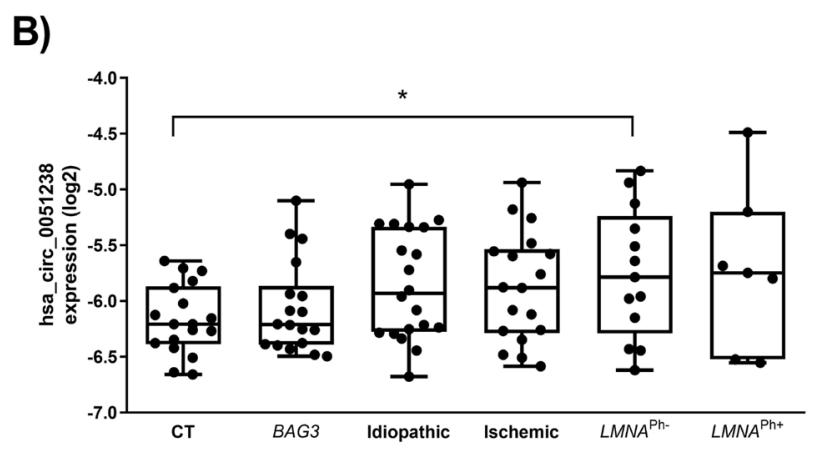

D)

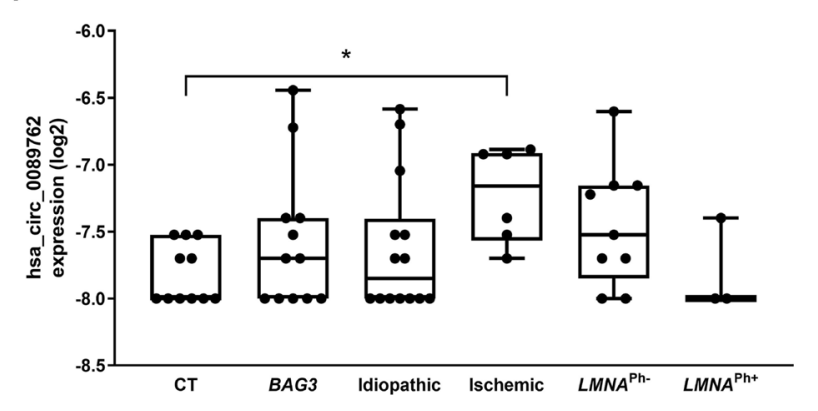

athanogene 3; CT, healthy cohort; circRNA, circular RNA; DCM, dilated cardiomyopathy; $L M N A$, lamin $\mathrm{A} / \mathrm{C} ; L M N A^{\mathrm{Ph}-}, L M N A$ carrier of the pathogenic variant; $L M N A^{\mathrm{Ph}+}, L M N A$ carrier phenotype positive

\section{Functional enrichment}

Information about circRNAs is available on the circBase website (http://www.circbase.org/). The Circular RNA Interactome (https://circinteractome.nia.nih.gov/) was used to predict miRNAs and RBP-binding sites. The regulatory network was performed with Navigator software [14]. The set of RBPs common to all the differentially expressed circRNAs was analyzed with STRING: functional protein association networks (https://string-db. org) [15]. The set of miRNAs common to all the differentially expressed circRNAs was analyzed with miRNet 2.0 (https://www.mirnet.ca/miRNet/home.xhtml).
Table 3 Comparisons of single circRNA as predictors of DCM

\begin{tabular}{llllll}
\hline DCM etiology & circRNA & AUC (95\% CI) & Sensitivity (\%) & Specificity (\%) & $p$ \\
\hline LMNA & hsa_circ_0003258 & $0.75(0.56-0.94)$ & 61.53 & 78.57 & 0.043 \\
& hsa_circ_0051238 & $0.71(0.53-0.88)$ & 70 & 72.73 & 0.02 \\
& hsa_circ_0051239 & $0.73(0.61-0.93)$ & 83.33 & 72.23 & 0.007 \\
Ischemic & hsa_circ_0089762 & $0.92(0.77-1)$ & 83.33 & 72.73 & 0.006 \\
\hline
\end{tabular}

$A U C$ area under the curve, $C I$ confidence interval, DCM dilated cardiomyopathy, LMNA lamin $\mathrm{A} / \mathrm{C}$ 


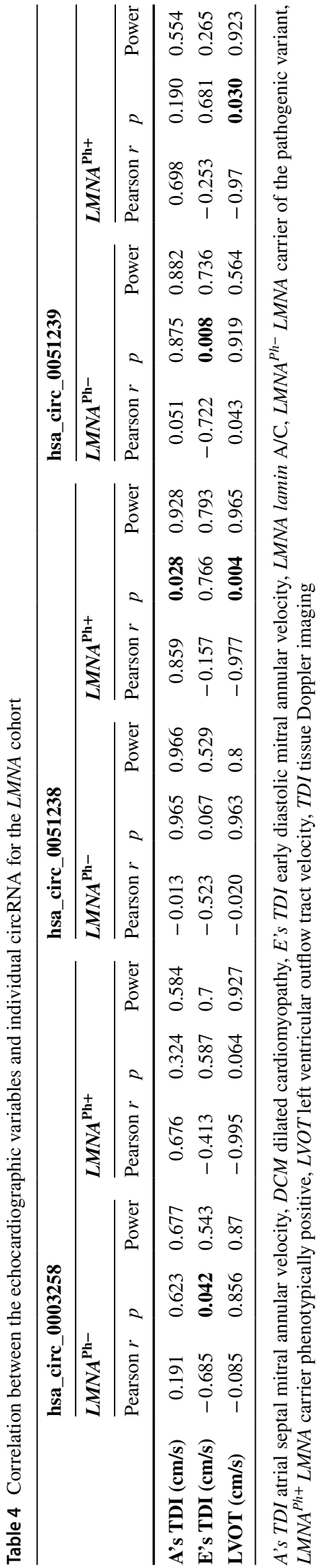

Fig. 3 Bivariate logistic regression analysis for $L M N A$-related DCM and ischemic DCM patients. A-F Logistic regression analysis for the LMNA-related DCM cohort. LVEF was independently negatively related with hsa_circ_0003258 (A), hsa_circ_0051238 (B), and hsa_circ_0051239 (C). D RV tricuspid annular plane systolic excursion (TAPSE) was negatively related to hsa_circ_0003258. E, F LV mitral annular plane systolic excursion (MAPSE) was negatively correlated with hsa_circ_0003258 and hsa_circ_0051238. G, H The levels of hsa_circ_0089762 were associated with A's TDI (G) and RV (H). The odds ratio, $95 \%$ of $\mathrm{CI}$, and $p$ values are indicated for each logistic regression analysis. Abbreviations: A's TDI, atrial septal mitral annular velocity; AUC, area under the curve; CT, healthy group; CI, confidence intervals; $L M N A$, lamin $\mathrm{A} / \mathrm{C}$ gene; $\mathrm{LVEF}$, left ventricle ejection fraction; MAPSE, mitral annular plane systolic excursion; OR, odd ratio; $\mathrm{RV}$; right ventricle; TAPSE, tricuspid annular plane systolic excursion

\section{Statistical analysis}

Continuous variables are expressed as the mean \pm standard deviation. Categorical variables are expressed in frequency and percentage (\%). Analysis of variance was applied to compare intergroup circRNAs levels. The Pearson correlation was used to test the link between echocardiographic and clinical variables vs. $\log _{2}$ circRNAs. In addition, the association between circRNAs and echocardiography parameters was assessed using logistic bivariate regression. Several models were constructed using the Wilcoxon test and iterating combinations between our circRNA candidates, as well as echocardiographic and clinical covariates. The changes in $p$-values of their variables were evaluated by the Wald test and a likelihood ratio. To characterize the diagnostic performance of the circRNAs candidate, ROC curves were applied together with a logistic regression model to determine the AUC and the specificity and sensitivity of the optimal cutoffs. ROC curves were generated by plotting sensitivity against 100 -specificity. Data were presented as the AUC and 95\% CI. The statistical software package $\mathrm{R}$ (www.r-project.org) was used for all analyses.

\section{Results}

\section{Analysis of circRNA expression profiles in plasma of DCM patients}

A total of 36 idiopathic and non-idiopathic DCM agematched patients were assessed to test the differences in circRNA expression profiles (see Supplementary Figs. 1

Table 5 Correlation between the clinical parameters and hsa circ_0089762 for ischemic DCM cohort

\begin{tabular}{llll}
\hline & \multicolumn{2}{l}{ hsa_circ_0089762 } & \\
\cline { 2 - 4 } & Pearson $r$ & $p$ & Power \\
\hline DBP (mm Hg) & -0.84 & 0.036 & 0.556 \\
LVEF (\%) & -0.842 & 0.036 & 0.561 \\
\hline
\end{tabular}

$D C M$ dilated cardiomyopathy, $D B P$ diastolic blood pressure, $L V E F$ left ventricle ejection fraction 
A) $\quad \mathrm{CT} \bullet \quad \angle M N A \mathrm{DCM}$

OR (95\% Cl): $0.81(0.68-0.98)$

$$
p=0.041
$$

AUC: 0.90 ; Sensitivity: 0.86

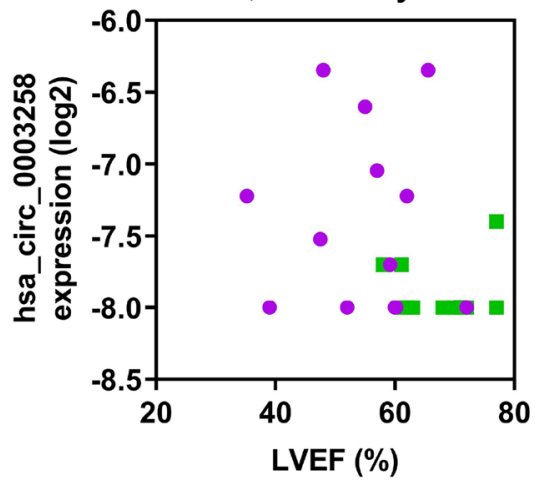

D)

- CT - LMNA DCM

OR (95\% CI): $0.69(0.48-0.99)$

$$
p=0.018
$$

AUC: 0.84 ; Sensitivity: 0.92

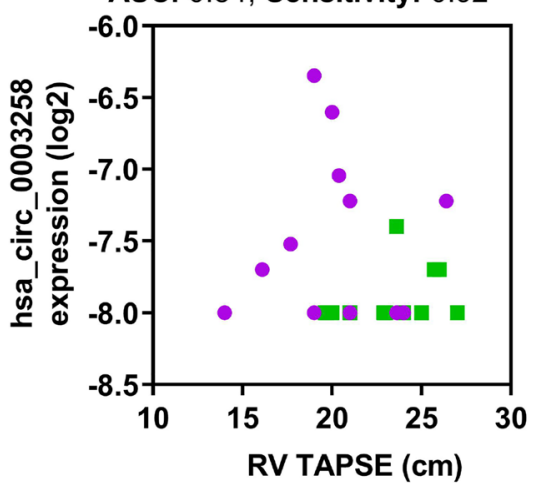

B) $\begin{aligned} & \text { CT } \bullet \text { LMNA DCM } \\ & \text { OR (95\% Cl): } 0.79(0.67-0.92) \\ & p<0.001\end{aligned}$

AUC: 0.92 ; Sensitivity: 0.83

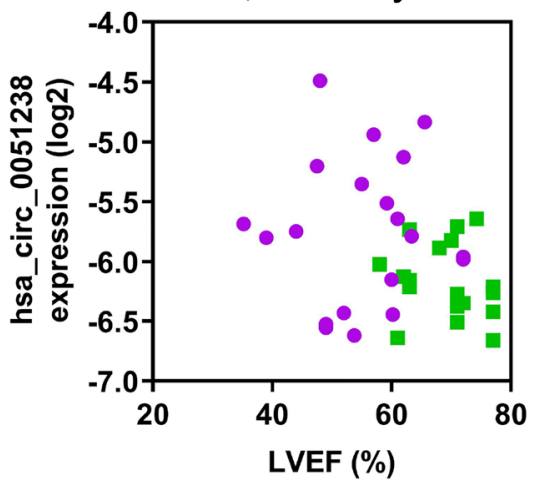

E)

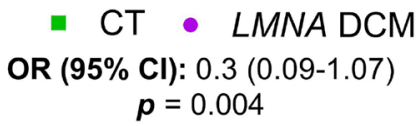

AUC: 0.95 ; Sensitivity: 0.93

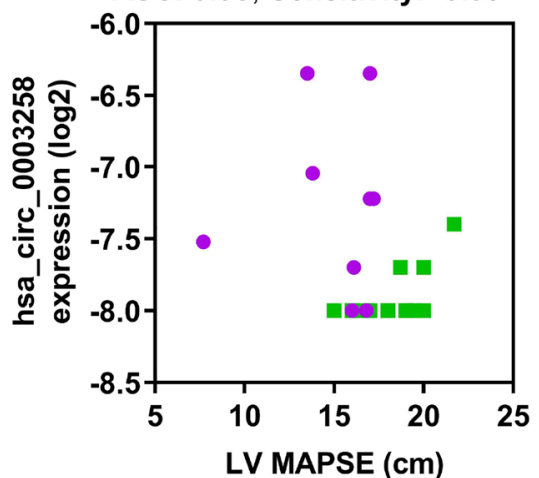

C)

- CT - LMNA DCM OR (95\% Cl): $0.8(0.69-0.94)$ $p<0.001$

AUC: 0.91 ; Sensitivity: 0.83

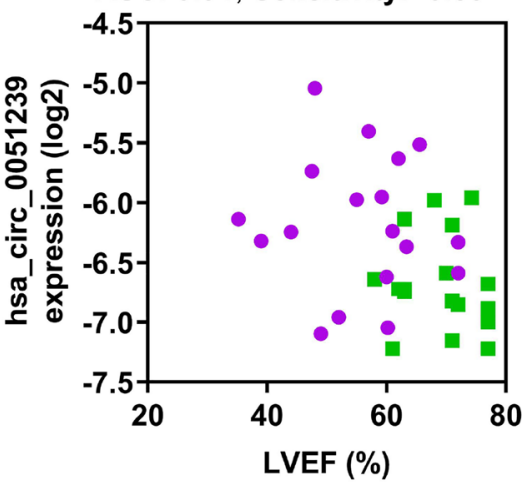

F)

- CT - LMNA DCM OR (95\% Cl): $0.53(0.32-0.89)$ $p<0.001$

AUC: 0.89 ; Sensitivity: 0.83

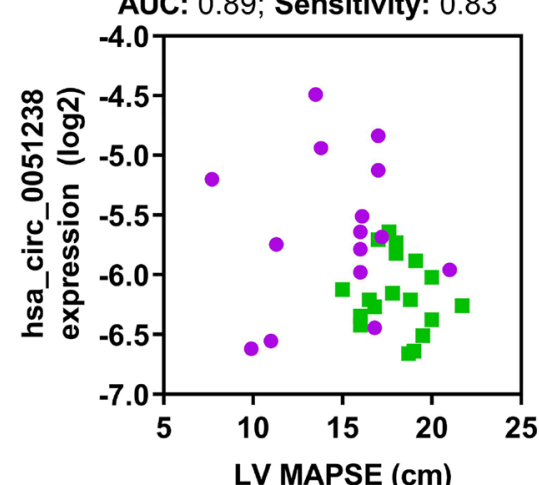

G) $\quad \mathrm{CT} \cdot \mathrm{Ischemic} \mathrm{DCM}$ OR (95\% Cl): 1.23 (0-9.71) $p=0.014$

AUC: 0.98 ; Sensitivity: 0.89

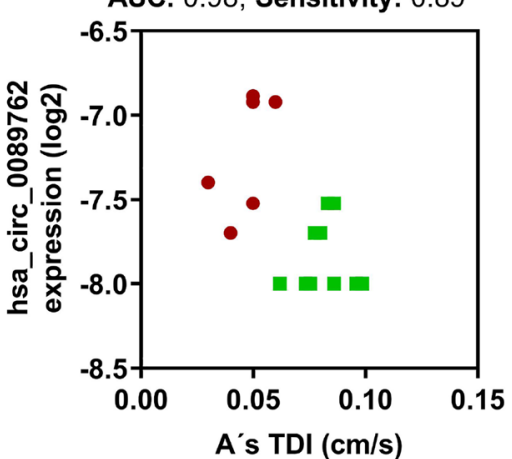

H) $\quad \mathrm{CT}$ - Ischemic DCM OR (95\% Cl): 2.85 (0.52-15.79) $p=0.029$

AUC: 0.96 ; Sensitivity: 0.9

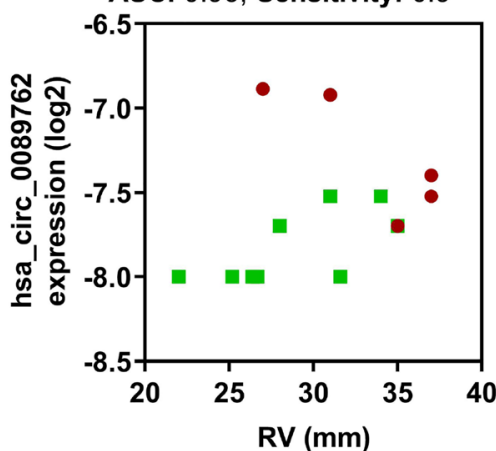


and 2). A total of ten candidate circRNAs (see Supplementary Table 2) were obtained from circRNA microarray screening of plasmatic samples (fold change $>2$, $p<0.05)$.

\section{Validating the expression of the candidate circRNAs}

The expression of the ten circRNA candidates was carried out in plasma samples of each study group, using qRT-PCR. Only LMNA and ischemic DCM populations showed differential circRNA expression (Table 2). Circulating levels of hsa_circ_0051238, hsa_circ_0051239, and hsa_circ_0003258 were highly upregulated in the LMNA population compared to healthy controls (Fig. 2). To assess the strength of circRNAs as an early biomarker before the clinical manifestation of malignant ventricular arrhythmias and LV dilation, the LMNArelated DCM group was subdivided into LMNA pathogenic variant carrier, phenotypically negative $\left(L M N A^{\mathrm{Ph}-}\right)$ and phenotypically positive $\left(L M N A^{\mathrm{Ph}+}\right)$. Circulating hsa_circ_0003258 levels were differentially expressed in the $L M N A^{\mathrm{Ph}-}\left(L M N A^{\mathrm{Ph}-} p=0.03, L M N A^{\mathrm{Ph}+} p=0.03\right)$
(Fig. 2A). The hsa_circ_0051238 levels were differentially expressed in the $L M N A^{\mathrm{Ph}-}$ population $(p=0.03)$ (Fig. 2B). And, the hsa_circ_0051239 plasmatic levels were significantly higher in both $L M N A$ groups $\left(L M N A^{\mathrm{Ph}-} p=0.03, L M N A^{\mathrm{Ph}+} p=0.04\right)$ than in healthy subjects (Fig. 2C). Regarding the ischemic DCM cohort, the plasma hsa_circ_0089762 levels were significantly higher $(p=0.04)$ than in healthy subjects (Fig. 2D).

\section{Diagnostic value of the validated circRNAs in a DCM population}

The receiver operating characteristic (ROC) area under the curve (AUC) analysis was assessed to investigate the circulating circRNAs diagnostic value in discriminating $L M N A$ and ischemic DCM etiology from healthy controls. All individual circRNAs show an AUC $\geq 0.7$. The highest AUC values reached by hsa_circ_0089762 that demonstrated an AUC value of 0.92 (95\% of confidence intervals $[\mathrm{CI}]$ range of specificities are shown in Table 3).

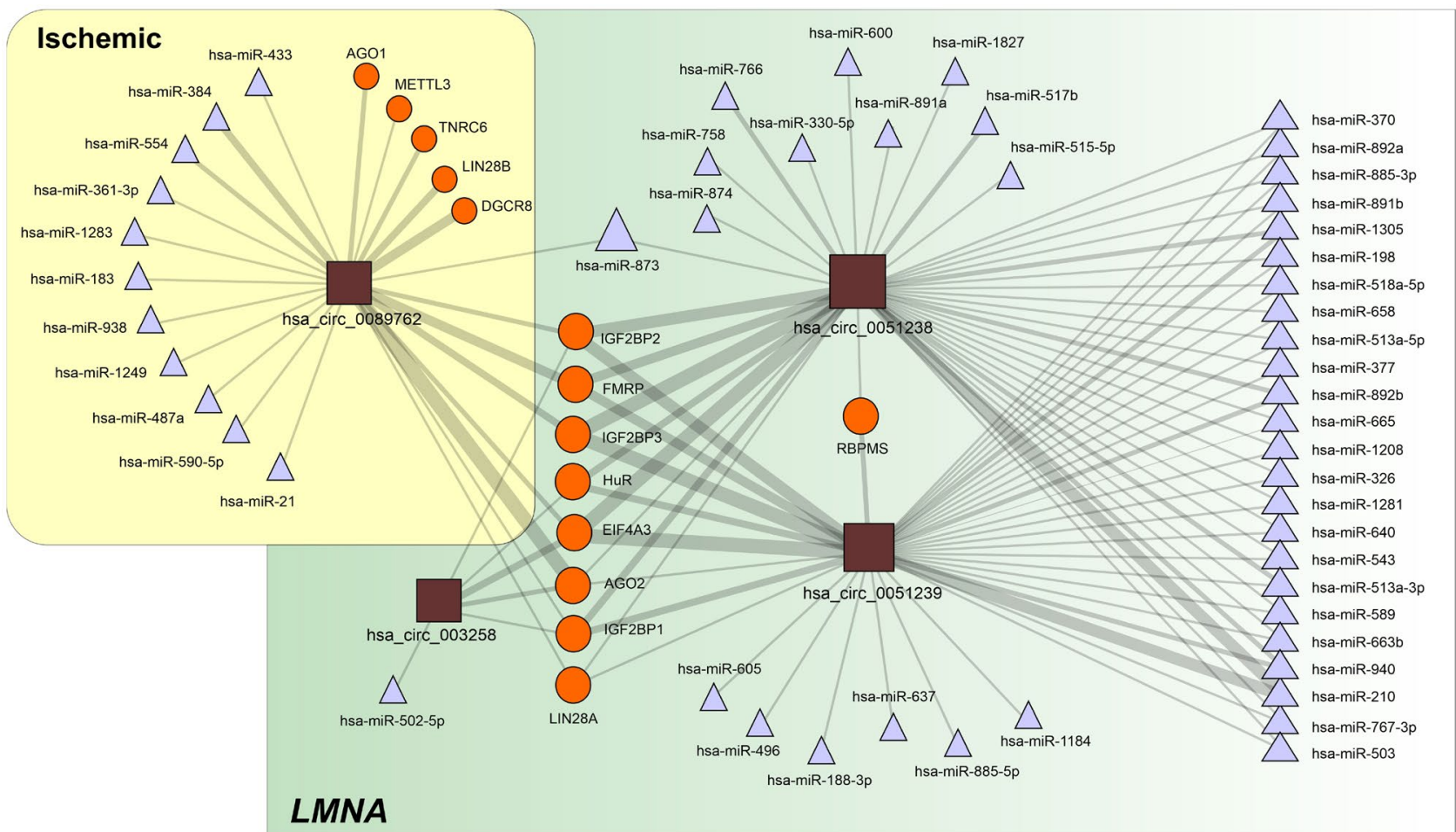

Fig. 4 CircRNA-centered regulatory network established among the selected circRNAs. The depicted interactions are based on data extracted from the circInteractome database and include miRNAs and RBPs. CircRNAs are represented as squares, RBPs as circles and miRNAs as triangles. The size of each symbol is proportional to the number of interactions established. The edge thickness is also propor- tional to the number of targets for each interacting partner as included in the circInteractome database. The regulatory network was prepared with Navigator software [14]. Abbreviations: DCM, dilated cardiomyopathy; LMNA, lamin A/C gene; miRNA, microRNA; RBP, RNAbinding protein 
Table 6 Differentially expressed circRNA potentially interact with RBP and miRNAs

\begin{tabular}{|c|c|c|c|}
\hline DCM etiology & circRNA & RBP & Predicted miRNAs target \\
\hline \multirow[t]{3}{*}{$L M N A$} & hsa_circ_003258 & AGO2, EIF4A3, HuR, IGF2BP1, IGF2BP2 & hsa-miR-502-5p \\
\hline & hsa_circ_0051238 & $\begin{array}{l}\text { AGO2, EIF4A3, FMRP, HuR, IGF2BP1, IGF2BP2, } \\
\text { IGF2BP3, LIN28A, RBPMS, ZC3H7B }\end{array}$ & $\begin{array}{l}\text { hsa-miR-1208, hsa-miR-1281, hsa-miR-1305, } \\
\text { hsa-miR-1827, hsa-miR-198, hsa-miR-210, } \\
\text { hsa-miR-326, hsa-miR-330-5p, hsa-miR-370, hsa- } \\
\text { miR-377, hsa-miR-503, hsa-miR-513a-3p, hsa- } \\
\text { miR-513a-5p, hsa-miR-515-5p, hsa-miR-517b, } \\
\text { hsa-miR-518a-5p, hsa-miR-543, hsa-miR-589, } \\
\text { hsa-miR-600, hsa-miR-640, hsa-miR-658, } \\
\text { hsa-miR-663b, hsa-miR-665, hsa-miR-758, } \\
\text { hsa-miR-766, hsa-miR-767-3p, hsa-miR-873, } \\
\text { hsa-miR-874, hsa-miR-885-3p, hsa-miR-891a, } \\
\text { hsa-miR-891b, hsa-miR-892a, hsa-miR-892b, } \\
\text { hsa-miR-940 }\end{array}$ \\
\hline & hsa_circ_0051239 & $\begin{array}{l}\text { AGO2, EIF4A3, FMRP, HuR, IGF2BP1, IGF2BP2, } \\
\text { IGF2BP3, LIN28A, RBPMS }\end{array}$ & $\begin{array}{l}\text { hsa-miR-1184, hsa-miR-1208, hsa-miR-1281, } \\
\text { hsa-miR-1305, hsa-miR-188-3p, hsa-miR-198, } \\
\text { hsa-miR-210, hsa-miR-326, hsa-miR-370, hsa- } \\
\text { miR-377, hsa-miR-496, hsa-miR-503, hsa-miR- } \\
\text { 513a-3p, hsa-miR-513a-3p, hsa-miR-513a-5p, } \\
\text { hsa-miR-518a-5p, hsa-miR-543, hsa-miR-589, } \\
\text { hsa-miR-605, hsa-miR-637, hsa-miR-640, hsa- } \\
\text { miR-658, hsa-miR-663b, hsa-miR-665, hsa- } \\
\text { miR-767-3p, hsa-miR-885-3p, hsa-miR-885-5p, } \\
\text { hsa-miR-891b, hsa-miR-892a, hsa-miR-892b, } \\
\text { hsa-miR-940 }\end{array}$ \\
\hline Ischemic & hsa_circ_0089762 & $\begin{array}{l}\text { AGO1, AGO2, DGCR8, EIF4A3, FMRP, IGF2BP1, } \\
\text { IGF2BP2, IGF2BP3, LIN28A, LIN28B, METTL3, } \\
\text { TNRC6 }\end{array}$ & $\begin{array}{l}\text { hsa-miR-183, hsa-miR-21, hsa-miR-361-3p, hsa- } \\
\text { miR-433, hsa-miR-590-5p }\end{array}$ \\
\hline
\end{tabular}

$D C M$ dilated cardiomyopathy, LMNA lamin A/C, RBP RNA-binding protein

\section{Association between the expression of circRNAs and the clinical characteristics of the DCM population}

The association between circulating circRNAs and echocardiographic and clinical features of DCM patients was also analyzed. As indicated in Table 4, the $L M N A^{\mathrm{Ph}-}$ group showed a negative correlation between hsa_circ_0003258 and hsa_circ_0051239 with early diastolic mitral annular velocity (E's TDI). The $L M N A^{\mathrm{Ph}+}$ cohort showed a positive correlation of hsa_circ_0051238 with tissue Doppler imaging (TDI) septal atrial systolic mitral annular velocity (A's TDI) and a negative correlation of hsa_circ_0051238 and hsa_circ_0051239 with LV outflow tract (LVOT) velocity.

An additional study was performed to assess correlations between the echocardiographic and clinical

Table 7 Pathway analysis main findings: PPI enrichment analysis

\begin{tabular}{lllll}
\hline Pathway & Overlap & FDR & Genes & Database \\
\hline Regulation of translation & $7 / 327$ & $2.09 \mathrm{e}-10$ & $A G O 2, E I F 4 A 3, E L A V L 1, F M R P, I G F 2 B P 1, I G F 2 B P 2, I G F 2 B P 3$ & $G O$ \\
mRNA binding & $7 / 198$ & $1.31 \mathrm{e}-12$ & $A G O 2, E I F 4 A 3, E L A V L 1, F M R P, I G F 2 B P 1, I G F 2 B P 2, I G F 2 B P 3$ & $G O$ \\
$\begin{array}{l}\text { Negative regulation of nitrogen com- } \\
\quad \text { pound metabolic process }\end{array}$ & $7 / 2307$ & $8.81 \mathrm{e}-06$ & $A G O 2, E I F 4 A 3, E L A V L 1, F M R P, I G F 2 B P 1, I G F 2 B P 2, I G F 2 B P 3$ & $G O$ \\
Regulation of mRNA stability & $5 / 113$ & $1.05 \mathrm{e}-08$ & ELAVL1,FMRP,IGF2BP1,IGF2BP2,IGF2BP3 & $G O$ \\
mRNA transport & $5 / 148$ & $3.12 \mathrm{e}-08$ & EIF4A3,FMRP,IGF2BP1,IGF2BP2,IGF2BP3 & $G O$ \\
Regulation of gene silencing by miRNA & $3 / 78$ & $3.82 \mathrm{e}-05$ & $A G O 2, E L A V L 1, F M R P$ & $G O$ \\
Regulation of membrane potential & $2 / 408$ & 0.0445 & EIF4A3,FMRP & $G O$ \\
MAPK6/MAPK4 signaling & $2 / 86$ & 0.0077 & $A G O 2, I G F 2 B P 1$ & $G O$ \\
ncRNA processing & $2 / 340$ & 0.0344 & $A G O 2, E I F 4 A 3$ & $G O$ \\
\hline
\end{tabular}

PPI enrichment $p$-value: $1.67 \mathrm{e}-10$

$F D R$ false discovery rate, $G O$ gene ontology, $K W$ keyword 
variables and hsa_circ_0089762 for the ischemic DCM population. Hsa_circ_0089762 expression was negatively associated with diastolic blood pressure and LVEF (see Table 5).

To further explore the expression of circRNA-DCM disease association, a logistic regression analysis was carried out in our DCM population (Fig. 3). All three LMNA-linked circRNAs were significantly related to male gender hsa_circ_0003258, hsa_circ_0051238, and hsa_ circ_0051239. In the LMNA cohort, the bivariate logistic regression analyses revealed that all LVEF were independently negatively associated with hsa_circ_0003258, hsa circ_0051238, and hsa_circ_0051239. LV mitral annular plane systolic excursion was independent negatively associated with hsa_circ_0003258 and hsa_circ_0051238. Right ventricle (RV) tricuspid annular plane systolic excursion was only independently negatively associated with hsa_circ_0003258. Pulmonary hypertension (PHT) was independently positively related to hsa_circ_0003258 and hsa_circ_0051239.

In the case of hsa_circ_0089762, the logistic regression analysis showed that its circulating levels within A's TDI wave or the RV dimension were independent influencing factors for ischemic DCM.

\section{Annotation for circRNA/RBPs interaction}

An examination of biological processes related to RBPs, with binding sites for circRNA candidates, reveals a set of possible pathways in which circRNAs play a regulative role. LMNA mutation influences the proper development of megakaryocytes resulting in altered platelet production/function [16]. We recovered this LMNA effect in the enrichment (GO:0,045,652), regulation of megakaryocyte differentiation $(F D R=0.0013)$, and fibroblast growth $(\mathrm{GO} 0,008,543)(\mathrm{FDR}=0.0267)$.

The analysis of the intersection set of RBPs predicted to interact with the selected circRNAs (Fig. 4; Table 6) shows clear enrichment in proteins involved in the control of transcriptional and translational processes (Table 7). Note the association with the regulation of membrane potential, in which IEF4A3 and FMRP are involved $(\mathrm{FDR}=0.045)$.

The analysis of miRNAs sponged by validated circRNAs offers various candidates for further research. Hsa_circ_0003258 has only one functional binding site to hsa-miR-653. As a counterpart, hsa_circ_0051238 and hsa_circ_0051239 present a clear sponge effect over hsa-miR-210, with five binding sites that have $\Delta U$ below zero. Thereby, the overexpression of hsa_circ_0051238 and hsa_circ_0051239 will actively reduce the availability of hsa-miR-210. Hsa-miR-210 regulates expression of hepatocyte growth factor gene, whose overexpression is considered a treatment for DCM [17]. Additionally, they also present a functional binding site for hsa-miR-330-5p that is involved in cardiomyocyte survival and function recovery [18]. Regarding miRNA-related diseases, hsa_circ_0051238 sponges hsa-miR-873 and hsa-miR$513 \mathrm{a}-5 \mathrm{p}$ are both related with heart disease $(p=0.075)$, and hsa-miR-377 is related with ischemic cardiomyopathy $(p=0.221)$. Hsa_circ_0089762 has sponge activity with multiple, energetically favorable binding sites. Of note is hsa-miR-21, as well as hsa-miR-183, hsa-miR-361-3p, hsa-miR-384, hsa-miR-873, hsa-miR-938, hsa-miR-1249, and hsa-miR-1283. The miRNAs sponged by the circRNAs with a context score over $90 \%$ was used to capture the set of mRNAs regulated by these miRNAs. Functional enrichment, using a hypergeometric association algorithm, shows that 148 proteins of the network were related with focal adhesion $\left(p=2.68 \mathrm{e}^{-8}\right)$, and 128 proteins were linked with regulation of the actin cytoskeleton $(p=0.00002)$. Gene ontology biological processes, using the same hypergeometric algorithm, show a significant correlation with endoplasmic reticulum-nuclei signaling pathways $\left(p=0.1 \mathrm{e}^{-6}\right)$ and pre- and post-Golgi vesicle transportation $\left(p=8.6 \mathrm{e}^{-7}\right.$ and $4.47 \mathrm{e}^{-7}$, respectively).

\section{Discussion}

Over the last decade, the diagnostic process of DCM etiologies has focused on searching for new biomarkers. An efficient biomarker for DCM should be robust, stable, non-invasive, sensitive, specific to this entity, predictive of a particular DCM etiology, and show a preclinical and clinical relevance to be validated in animal and/or human cell models [19]. We propose the use of peripheral circRNAs as a novel discriminant biomarker of DCM etiologies.

Unlike linear RNA, single circulating circRNAs or circRNAs combined with various other biomarkers are a promising tool for clinical diagnosis of heart diseases, which would improve outcome [20]. Thus, circRNA MICRA was reported to risk-stratify patients after acute myocardial infarction [21]. Peripheral circ_0124644 and circ_0098964 levels have been described as a diagnostic biomarker of coronary artery disease [22]. Related to cardiomyopathies, a set of circulating circRNAs DNAJC6, TMEM56, and MBOAT2 has been proposed to discriminate between healthy and hypertrophic cardiomyopathy [23]. In this sense, hsa_circ_0071542 was upregulated in children with fulminant myocarditis in leukocytes isolated from peripheral blood [24]. Nevertheless, this area remains mostly unexplored in DCM $[22,25]$. Recent studies have described several circRNA expression profiles in the DCM 
population compared to healthy patients. However, to date, it has not been studied among the different etiologies of DCM [26, 27]. Hence, further analysis of circRNAs among DCM etiologies might provide early, precise characterization of the disease and lead to novel pathological information, beyond the traditional biomarkers. To the best of our knowledge, the present study is the first to describe a subset of circulating circRNA for a discriminative etiology-based diagnostic in DCM. Circulating hsa_circ_0003258, hsa_ circ_0051238, and hsa_circ_0051239 expression levels were upregulated in $L M N A$-related DCM patients. Notably, hsa_circ_0051238 plasmatic levels were significantly present in the $L M N A^{\mathrm{Ph}-}$ cohort. Hence, it may be a promising diagnostic biomarker for the early identification of patients in an initial stage of $L M N A$-related DCM. This will allow personalized therapeutic measures to be applied that help to improve the progression and outcome of LMNArelated DCM. Furthermore, plasmatic hsa_circ_0089762 may provide discriminative power for the ischemic DCM cohort with high-yield diagnostic accuracy and an AUC of 0.92 . These circRNAs have been identified mostly in various types of oncologic processes [28-33]. Thus, only hsa_circ_0051239 levels have been upregulated in the myocardium of congenital ventricular septal defect [33]. However, they have not been previously described in DCM cases.

In the current study, circRNA were related to clinical and echocardiographic variables. Male gender, rare nonmissense variants in LMNA and LVEF $<50 \%$ have been established as independent factors associated with a more aggressive outcome and even death during follow-up [34]. Herein, all three circRNAs associated with LMNA-DCM etiology were related to male gender [35]. On the other side, echocardiography variables and related circRNAs might suggest a time-evolving sequence. TDI echocardiography is a non-invasive, very sensitive method to assess the cardiac hemodynamic in DCM [36]. TDI reveals that subtle impairments in diastolic myocardial tissue velocities are markers of early cardiac disease and have been associated with outcome in various cardiopathies $[16,17]$. In the $L M N A^{\mathrm{Ph}-}$ group, the E's TDI is negatively related to hsa_circ_0003258 and hsa_circ_0051239. This E's TDI impairment suggests an underlying early diastolic dysfunction [37]. A's TDI in the $L M N A^{\mathrm{Ph}+}$ group showed a positive correlation, which indicates that the left atrium is a prominent factor to maintain the LV filling pressure when diastolic dysfunction advances. This sequential TDI septal impairment mirrors the transition from $L M N A^{\mathrm{Ph}-}$ to $L M N A^{\mathrm{Ph}+}$ and may be related to the progressive fibrosis of the interventricular septum located in the basal portion, which is characteristic of the LMNA relatedDCM that has been associated with ventricular arrhythmias and worse prognosis [38]. LVEF was independently negatively associated with hsa_circ_0003258, hsa_circ_0051238, and hsa_circ_0051239. According to the LV systolic impairment, hsa_circ_0003258 and hsa_circ_0051238 were related to LV mitral annular plane systolic excursion. Thus, changes in contractility quantified by LV mitral annular plane systolic excursion occur as compensatory mechanisms before impairment of ventricular function [39]. Hsa_circ_0051238 and hsa_circ_0051239 were also negatively related to LVOT velocity, which suggests progressive impairment of the cardiac pump in the $L M N A^{\mathrm{Ph}+}$ cohort. Dysfunction of $\mathrm{RV}$ is a final common step in DCM and heart failure [40]. RV pressure overload due to PHT and the interventricular interdependence affected by septal fibrosis and underlying ischemia may influence this situation. In support of our results, circRNA, hsa_circ_0003258 was positively increased with the $\mathrm{RV}$ lower tricuspid annular plane systolic excursion and PHT [41].

Otherwise, hsa_circ_0089762 correlated to diastolic blood pressure and LVEF in the ischemic group, which supports our results as a specific, highly sensitive biomarker with high-yield diagnostic accuracy. Moreover, hsa_circ_0089762 was related to A's TDI, which suggests more advanced progression of this entity. Its association with an increase in RV dimension could add information for tailored management in this group, since RV impairment is a worse outcome marker in the ischemic population [42]. In addition, RV involvement has a multifactorial origin that may be influenced by LV remodelling, increased LV filling pressures, and the appearance of PHT or RV ischemia [43].

Regarding biological implications, circRNAs spring from introns or exons of their parental genes by backspliced circularization [25]. Hence, the ratio between linear and circular fractions affects gene expression. According to the protein atlas (proteinatlas.org), parental genes are expressed in cardiac tissue, which supports correlations between etiologies and circRNAs. Hsa_circ_0003258 is synthesized from ZNF652 gene. ZNF652 interacts with CBFA2T3, which acts as a transcriptional repressor [44]. ZNF652 is associated with systolic or diastolic blood pressure and hypertension. However, its role remains unclear [45]. Hsa_circ_0051238 and hsa_circ_0051239 come from the ATP5SL gene. ATP5SL is required for the assembly of mitochondrial NADH: ubiquinone oxidoreductase complex (complex I). Complex I is essential to provide the energy for cardiac function and is related to DCM progression [46]. ATP5SL has been associated with a congenital ventricular septal defect by the overexpression of hsa_circ_0051239 [47]. Finally, hsa_circ_0089762 is generated from the MT-CO2 gene. MT-CO2 is part of the electron transport chain of the mitochondria. Reduced activity of the electron transport chain subunits has been described independently of etiology in ischemic or idiopathic DCM patients [48]. 
The functional enrichment of the intersecting set of RBSs reveals the role of FMRP in regulation of the membrane potential. Bao et al. described FMRP isoform 1, in rats, as an essential protection factor and a novel potential biomarker in the cardiovascular system [49]. The participation of circRNAs in regulatory networks involving competing-endogenous RNA interactions by sequestering miRNAs has been characterized recently in cardiovascular pathologies $[50,51]$. From the set of miRNAs that could be sponged by the circRNAs that we considered, we found significant enrichment in the regulation of focal adhesion and actin cytoskeleton. Both have an important role in human DCM [52], which suggests new pathways of study.

Our current study has several limitations. Firstly, our sample was prospectively recruited from the outpatient clinic. The size of the study sample, comprised of strictly DCM patients, did not allow us to obtain a robust multivariate logistic regression model. Furthermore, a larger sample size is needed to validate these data by gender categorization since gender may play a role in the DCM prognosis $[53,54]$. In consequence, these results should be extended and replicated to a larger population before the novel biomarkers can be routinely applied in clinical practice. Furthermore, data on natriuretic peptides or troponin were not accessible for all patients. Finally, even though databases registered the expression of the parental genes in cardiac tissue, we have no confirmation about the direct secretion from the heart of these circulating circRNAs into the extracellular space. Hence, the association of circRNAs with DCM and all the interactions are putative. Further analysis should be carried out on human heart samples to confirm our results.

\section{Conclusion}

Exploring new biomarkers through circular transcriptome expression patterns will identify new targets in DCM pathogenesis. We propose a circulating circRNAs fingerprint to discriminate between various DCM etiologies. Circulating hsa_circ_0003258, hsa_circ_0051238, and hsa_circ_0051239 expression levels are higher in $L M N A$-related DCM, and hsa_circ_0089762 levels are specifically upregulated in the ischemic DCM cohort. These circulating circRNAs and certain echocardiographic variables might improve the etiologybased diagnostic, which allows early identification of asymptomatic cases and tailored treatment of the DCM population.

\footnotetext{
Abbreviations A's TDI: Atrial septal mitral annular velocity; AUC : Area under the curve; BAG3: BCL2-associated athanogene 3; CI: Confidence interval; circRNA: Circular RNA; DCM: Dilated cardiomyopathy; E's TDI: Early diastolic mitral annular velocity; FDR: False discovery rate; $L M N A$ : Lamin A/C; LV: Left ventricular; LVEF: LV ejection fraction; LVOT: LV outflow tract; MAPSE
}

: Mitral annular plane systolic excursion; mRNA: Messenger RNA; miRNA: MicroRNA; PHT: Pulmonary hypertension; ROC: Receiver operating characteristic; RBP: RNA binding protein; qRT-PCR: Quantitative real-time polymerase chain reaction; TAPSE: Tricuspid annular plane systolic excursion; TDI: Tissue Doppler imaging

Supplementary Information The online version contains supplementary material available at https://doi.org/10.1007/s00109-021-02119-6.

Acknowledgements We would like to thank Galan Pacheco for statistical support. We would also like to thank all patients involved in this project.

Author contribution All authors have read and approved the submission of the manuscript. Calderon-Dominguez M, Mangas A, and Toro R conceived the experiments; Quezada-Feijoo M, Ramos M, Campuzano O, Sarquellas Brugada-G, Pinilla JM, Robles Mezcua A, Mangas A, and Toro R recruited the subjects; and Thalia extracted RNA from samples. Costa M, Calderon-Dominguez M, Pacheco-Cruz GA, Enguita FJ, and Toro R conducted the experiments and analyzed the results. CalderonDominguez M, Mangas A, and Toro R wrote the manuscript. All authors reviewed the manuscript.

Funding Open Access funding provided thanks to the CRUE-CSIC agreement with Springer Nature. This work was supported by grants in the framework of the European Regional Development Fund (ERDF) Integrated Territorial Initiative (ITI PI0048-2017 and ITI0033_2019), a clinical research grant from the Spanish Society of Cardiology for Basic Research in cardiology (PI0012_2019), COST (European Cooperation in Science and Technology) Action EUCardioRNA CA17129, and the Portuguese Foundation for Science and Technology (FCT) under the framework of the research grant PTDC-MED-GEN-29389-2017.

Data availability Data transparency is guaranteed. The datasets generated during and/or analyzed during the current study are available from the corresponding author on reasonable request.

Code availability We used various softwares for functional enrichment and statistical analysis. All of them are cited in our manuscript.

\section{Declarations}

Conflict of interests We know of no conflicts of interest associated with this publication, and there has been no significant financial support for this work that could have influenced its outcome. The study protocol was approved by the Andalusian Biomedical Research Ethics committee. The study was performed in full compliance with the Declaration of Helsinki. Informed consent was obtained from all subjects involved in the study. Written informed consent has been obtained from the patients for paper publication.

Open Access This article is licensed under a Creative Commons Attribution 4.0 International License, which permits use, sharing, adaptation, distribution and reproduction in any medium or format, as long as you give appropriate credit to the original author(s) and the source, provide a link to the Creative Commons licence, and indicate if changes were made. The images or other third party material in this article are included in the article's Creative Commons licence, unless indicated otherwise in a credit line to the material. If material is not included in the article's Creative Commons licence and your intended use is not permitted by statutory regulation or exceeds the permitted use, you will need to obtain permission directly from the copyright holder. To view a copy of this licence, visit http://creativecommons.org/licenses/by/4.0/. 


\section{References}

1. Savarese G, Lund LH (2017) Global public health burden of heart failure. Card Fail Rev 03:7. https://doi.org/10.15420/cfr.2016: $25: 2$

2. McNally EM, Mestroni L (2017) Dilated cardiomyopathy: genetic determinants and mechanisms. Circ Res 121:731-748. https://doi. org/10.1161/CIRCRESAHA.116.309396

3. Rosenbaum AN, Agre KE, Pereira NL (2020) Genetics of dilated cardiomyopathy: practical implications for heart failure management. Nat Rev Cardiol 17:286-297

4. Van Der Bijl P, Delgado V, Bootsma M, Bax JJ (2018) Risk stratification of genetic, dilated cardiomyopathies associated with neuromuscular disorders. Circulation 137:2514-2527. https://doi.org/ 10.1161/CIRCULATIONAHA.117.031110

5. Domínguez F, Cuenca S, Bilińska Z et al (2018) Dilated cardiomyopathy due to BLC2- associated athanogene 3 (BAG3) mutations. J Am Coll Cardiol 72:2471-2481. https://doi.org/10.1016/j. jacc.2018.08.2181

6. Kumar S, Baldinger SH, Gandjbakhch E et al (2016) Long-term arrhythmic and nonarrhythmic outcomes of lamin A/C mutation carriers. J Am Coll Cardiol 68:2299-2307. https://doi.org/10. 1016/j.jacc.2016.08.058

7. Lu D, Thum T (2019) RNA-based diagnostic and therapeutic strategies for cardiovascular disease. Nat Rev Cardiol 16:661-674

8. Memczak S, Papavasileiou P, Peters O, Rajewsky N (2015) Identification and characterization of circular RNAs as a new class of putative biomarkers in human blood. PLoS ONE 10:e0141214. https://doi.org/10.1371/journal.pone.0141214

9. Stepien E, Costa MC, Kurc S et al (2018) The circulating noncoding RNA landscape for biomarker research: lessons and prospects from cardiovascular diseases review-article. Acta Pharmacol Sin 39:1085-1099. https://doi.org/10.1038/aps.2018.35

10. Elliott P, Andersson B, Arbustini E et al (2008) Classification of the cardiomyopathies: a position statement from the european society of cardiology working group on myocardial and pericardial diseases. Eur Heart J 29:270-276. https://doi.org/10.1093/ eurheartj/ehm342

11. Belmonte T, Mangas A, Calderon-Dominguez $\mathrm{M}$ et al (2020) Peripheral microRNA panels to guide the diagnosis of familial cardiomyopathy. Transl Res 218:1-15. https://doi.org/10.1016/j. trs1.2020.01.003

12. Toro R, Blasco-Turrión S, Morales-Ponce FJ et al (2018) Plasma microRNAs as biomarkers for lamin A/C-related dilated cardiomyopathy. J Mol Med 96:845-856

13. Dudekula DB, Panda AC, Grammatikakis I et al (2016) Circinteractome: a web tool for exploring circular RNAs and their interacting proteins and microRNAs. RNA Biol 13:34-42. https://doi.org/ 10.1080/15476286.2015.1128065

14. Shirdel EA, Xie W, Mak TW, Jurisica I (2011) NAViGaTing the micronome - using multiple microRNA prediction databases to identify signalling pathway-associated microRNAs. PLoS ONE 6:e17429. https://doi.org/10.1371/journal.pone.0017429

15. Szklarczyk D, Gable AL, Lyon D et al (2019) STRING v11: protein-protein association networks with increased coverage, supporting functional discovery in genome-wide experimental datasets. Nucleic Acids Res 47:D607-D613. https://doi.org/10. 1093/nar/gky1131

16. Izquierdo I, Rosa I, Bravo SB et al (2016) Proteomic identification of putative biomarkers for early detection of sudden cardiac death in a family with a LMNA gene mutation causing dilated cardiomyopathy. J Proteomics 148:75-84. https://doi.org/10.1016/j. jprot.2016.07.020

17. Komamura K, Tatsumi R, Miyazaki J et al (2004) Treatment of dilated cardiomyopathy with electroporation of hepatocyte growth factor gene into skeletal muscle. Hypertens (Dallas, Tex 1979) 44:365-71. https://doi.org/10.1161/01.HYP.0000139916.96375. 47

18. Van Rooij E, Sutherland LB, Liu N et al (2006) A signature pattern of stress-responsive microRNAs that can evoke cardiac hypertrophy and heart failure. Proc Natl Acad Sci U S A 103:18255-18260. https://doi.org/10.1073/pnas.0608791103

19. Martín-Ventura JL, Blanco-Colio LM, Tuñón J et al (2009) Biomarkers in cardiovascular medicine. Rev Española Cardiol (English Ed 62:677-688. https://doi.org/10.1016/s1885-5857(09) 72232-7

20. Khan MAF, Reckman YJ, Aufiero $S$ et al (2016) RBM20 regulates circular RNA production from the titin gene. Circ Res 119:9961003. https://doi.org/10.1161/CIRCRESAHA.116.309568

21. Salgado-Somoza A, Zhang L, Vausort M, Devaux Y (2017) The circular RNA MICRA for risk stratification after myocardial infarction. IJC Hear Vasc 17:33-36. https://doi.org/10.1016/j.ijcha.2017.11.001

22. Zhao Z, Li X, Gao C et al (2017) Peripheral blood circular RNA hsa-circ-0124644 can be used as a diagnostic biomarker of coronary artery disease. Sci Rep 7:1-9. https://doi.org/10. 1038/srep39918

23. Sonnenschein K, Wilczek AL, de Gonzalo-Calvo D et al (2019) Serum circular RNAs act as blood-based biomarkers for hypertrophic obstructive cardiomyopathy. Sci Rep 9:1-8. https://doi. org/10.1038/s41598-019-56617-2

24. Zhang L, Han B, Wang J et al (2019) Differential expression profiles and functional analysis of circular RNAs in children with fulminant myocarditis. Epigenomics 11:1129-1141. https://doi. org/10.2217/epi-2019-0101

25. Gabriel AF, Costa MC Enguita FJ (2020) Circular RNA-centered regulatory networks in the physiopathology of cardiovascular diseases. Int J Mol Sci 21

26. Sun W, Han B, Cai D et al (2020) Differential expression profiles and functional prediction of circular RNAs in pediatric dilated cardiomyopathy. Front Mol Biosci 7:600170. https://doi.org/10. 3389/fmolb.2020.600170

27. Lin Z, Zhao Y, Dai F et al (2021) Analysis of changes in circular RNA expression and construction of ceRNA networks in human dilated cardiomyopathy. J Cell Mol Med 25:2572-2583. https:// doi.org/10.1111/jemm. 16251

28. Guo J, Duan H, Li Y et al (2019) A novel circular RNA circZNF652 promotes hepatocellular carcinoma metastasis through inducing snail-mediated epithelial-mesenchymal transition by sponging miR-203/miR-502-5p. Biochem Biophys Res Commun 513:812-819. https://doi.org/10.1016/j.bbrc.2019.03.214

29. Fu C, Lv R, Xu G et al (2017) Circular RNA profile of infantile hemangioma by microarray analysis. PLoS ONE 12:1-16. https:// doi.org/10.1371/journal.pone.0187581

30. WL Mo JT Jiang L Zhang et al 2020 Circular RNA hsa circ_0000467 promotes the development of gastric cancer by competitively binding to microRNA miR-326-3p. Biomed Res Int 2020 https://doi.org/10.1155/2020/4030826

31. Wu Z, Sun $\mathrm{H}$, Wang $\mathrm{C}$ et al (2020) Mitochondrial genome-derived circRNA mc-COX2 functions as an oncogene in chronic lymphocytic leukemia. Mol Ther - Nucleic Acids 20:801-811. https://doi. org/10.1016/j.omtn.2020.04.017

32. Yavropoulou M, Poulios C, Michalopoulos N et al (2018) A role for circular non-coding RNAs in the pathogenesis of sporadic parathyroid adenomas and the impact of gender-specific epigenetic regulation. Cells 8:15. https://doi.org/10.3390/cells8010015

33. Xu C, Yu Y, Ding F (2018) Microarray analysis of circular RNA expression profiles associated with gemcitabine resistance in pancreatic cancer cells. Oncol Rep 40:395-404. https://doi.org/10. 3892/or.2018.6450

34. Van Rijsingen IAW, Arbustini E, Elliott PM et al (2012) Risk factors for malignant ventricular arrhythmias in lamin $\mathrm{A} / \mathrm{C}$ mutation 
carriers: a European cohort study. J Am Coll Cardiol 59:493-500. https://doi.org/10.1016/j.jacc.2011.08.078

35. van Rijsingen IAW, Nannenberg EA, Arbustini E et al (2013) Gender-specific differences in major cardiac events and mortality in lamin A/C mutation carriers. Eur J Heart Fail 15:376-384. https://doi.org/10.1093/eurjhf/hfs191

36. Van Rijsingen IAW, Bakker A, Azim D et al (2013) Lamin A/C mutation is independently associated with an increased risk of arterial and venous thromboembolic complications. Int J Cardiol 168:472-477. https://doi.org/10.1016/j.ijcard.2012.09.118

37. Pérez-Serra A, Toro R, Campuzano O et al (2015) A novel mutation in lamin $\mathrm{A} / \mathrm{C}$ causing familial dilated cardiomyopathy associated with sudden cardiac death. J Card Fail 21:217-225. https:// doi.org/10.1016/j.cardfail.2014.12.003

38. Fontana M, Barison A, Botto N et al (2013) CMR-verified interstitial myocardial fibrosis as a marker of subclinical cardiac involvement in LMNA mutation carriers. JACC Cardiovasc Imaging 6:124-126. https://doi.org/10.1016/j.jcmg.2012.06.013

39. Hernandez-Suarez DF, Lopez-Menendez F, Roche-Lima A, Lopez-Candales A (2019) Assessment of mitral annular plane systolic excursion in patients with left ventricular diastolic dysfunction. Cardiol Res 10:83-88. https://doi.org/10.14740/cr837

40. Rudski LG, Lai WW, Afilalo J et al (2010) Guidelines for the echocardiographic assessment of the right heart in adults: a report from the American Society of Echocardiography. Endorsed by the European Association of Echocardiography, a registered branch of the European Society of Cardiology, and. J Am Soc Echocardiogr 23:685-713

41. Merlo M, Gobbo M, Stolfo D et al (2016) The prognostic impact of the evolution of RV function in idiopathic DCM. JACC Cardiovasc Imaging 9:1034-1042. https://doi.org/10.1016/j.jcmg.2016. 01.027

42. Brieke A, DeNofrio D (2005) Right ventricular dysfunction in chronic dilated cardiomyopathy and heart failure. Coron Artery Dis 16:5-11. https://doi.org/10.1097/00019501-200502000-00002

43. Kukulski T, She L, Racine $N$ et al (2015) Implication of right ventricular dysfunction on long-term outcome in patients with ischemic cardiomyopathy undergoing coronary artery bypass grafting with or without surgical ventricular reconstruction. J Thorac Cardiovasc Surg 149:1312-1321. https://doi.org/10. 1016/j.jtcvs.2014.09.117
44. Kumar R, Selth LA, Schulz RB et al (2011) Genome-wide mapping of ZNF652 promoter binding sites in breast cancer cells. J Cell Biochem 112:2742-2747. https://doi.org/10.1002/jcb.23214

45. Korkor MT, Meng FB, Xing SY et al (2011) Microarray analysis of differential gene expression profile in peripheral blood cells of patients with human essential hypertension. Int J Med Sci 8:168 179. https://doi.org/10.7150/ijms.8.168

46. Jarreta D, Orús J, Barrientos A et al (2000) Mitochondrial function in heart muscle from patients with idiopathic dilated cardiomyopathy. Cardiovasc Res 45:860-865. https://doi.org/10.1016/ S0008-6363(99)00388-0

47. Liu H, Hu Y, Zhuang B et al (2018) Differential expression of CircRNAs in embryonic heart tissue associated with ventricular septal defect. Int J Med Sci 15:703-712. https://doi.org/10.7150/ijms.21660

48. Govindaraj P, Rani B, Sundaravadivel P et al (2019) Mitochondrial genome variations in idiopathic dilated cardiomyopathy. Mitochondrion 48:51-59. https://doi.org/10.1016/j.mito.2019. 03.003

49. Bao J, Ye C, Zheng Z, Zhou Z (2018) Fmr1 protects cardiomyocytes against lipopolysaccharide-induced myocardial injury. Exp Ther Med 16:1825-1833. https://doi.org/10.3892/etm.2018.6386

50. Wang K, Gan TY, Li N et al (2017) Circular RNA mediates cardiomyocyte death via miRNA-dependent upregulation of MTP18 expression. Cell Death Differ 24:1111-1120. https://doi.org/10. 1038/cdd.2017.61

51. Costa MC, Cortez-Dias N, Gabriel A et al (2019) circRNAmiRNA cross-talk in the transition from paroxysmal to permanent atrial fibrillation. Int J Cardiol 290:134-137. https://doi.org/10. 1016/j.ijcard.2019.04.072

52. Towbin JA (1998) The role of cytoskeletal proteins in cardiomyopathies. Curr Opin Cell Biol 10:131-139. https://doi.org/10.1016/ S0955-0674(98)80096-3

53. Yogasundaram H, Qi A, Nguyen Q, Oudit GY (2020) Battle of the sexes: differential prognosis by sex in dilated cardiomyopathy. Can J Cardiol 36:7-10

54. Cannatà A, Fabris E, Merlo M et al (2020) Sex differences in the long-term prognosis of dilated cardiomyopathy. Can J Cardiol 36:37-44. https://doi.org/10.1016/j.cjca.2019.05.031

Publisher's Note Springer Nature remains neutral with regard to jurisdictional claims in published maps and institutional affiliations.

\section{Authors and Affiliations}

\section{Marina C. Costa ${ }^{1}$ Maria Calderon-Dominguez ${ }^{2}$ - Alipio Mangas ${ }^{2,3,4} \cdot$ Oscar Campuzano $^{5,6,7}$. Georgia Sarquella-Brugada ${ }^{5,6,7}$. Mónica Ramos ${ }^{8} \cdot$ Maribel Quezada-Feijoo $^{8}$. José Manuel García Pinilla9, 10 . Ainhoa Robles-Mezcua ${ }^{9,10}$. Galan del Aguila Pacheco-Cruz ${ }^{2}$. Thalia Belmonte ${ }^{2}$. Francisco J. Enguita ${ }^{1} \cdot$ Rocío Toro $^{2,4}$}

\author{
Alipio Mangas \\ alipio.mangas@uca.es \\ Oscar Campuzano \\ oscar@brugada.org \\ Georgia Sarquella-Brugada \\ georgia@brugada.org \\ Mónica Ramos \\ monica.ramos81@gmail.com \\ Maribel Quezada-Feijoo \\ maribelquezada2000@gmail.com \\ Ainhoa Robles-Mezcua \\ ainhoa.mezcua@gmail.com
}

Galan del Aguila Pacheco-Cruz

marlucale41@gmail.com

Thalia Belmonte

thaliabelmonte@gmail.com

Francisco J. Enguita

fenguita@medicina.ulisboa.pt

1 Faculdade de Medicina, Instituto de Medicina Molecular João Lobo Antunes, Universidade de Lisboa, Av. Prof. Egas Moniz, 1649-028 Lisbon, Portugal

2 Biomedical Research and Innovation Institute of Cadiz (INiBICA), Research Unit, Puerta del Mar University Hospital, Cadiz, Spain 
3 Internal Medicine Department, Puerta del Mar University Hospital, Cadiz, Spain

4 Medicine Department, School of Medicine, University of Cádiz, Cadiz, Spain

5 Medical Science Department, School of Medicine, University of Girona, Girona, Spain

6 Cardiovascular Genetics Center, University of Girona-IDIBGI, Girona, Spain

7 Centro de Investigación Biomédica en Red, Enfermedades Cardiovasculares (CIBERCV), Madrid, Spain
8 Cardiology Department Hospital Cruz Roja, Alfonso X University, Madrid, Spain

9 Servicio de Cardiología, Unidad de Insuficiencia Cardíaca Y Cardiopatías FamiliaresHospital Universitario Virgen de La VictoriaIBIMA, Malaga, Spain

10 Consumo Y Bienestar Social, CIBER-Cardiovascular, Instituto de Salud Carlos III, Ministerio de Sanidad, Madrid, Spain 